\title{
Sensitivity estimates for diffuse, point-like and extended neutrino sources with KM3NeT/ARCA
}

\section{B. Caiffi ${ }^{a}$, A. Garcia Soto ${ }^{b, c}$, A. Heijboer ${ }^{b, c}$, V. Kulikovskiy ${ }^{a}$, R.S. Muller ${ }^{b, c, *}$ and M. Sanguineti $^{a, d}$ on behalf of the KM3NeT Collaboration}

(a complete list of authors can be found at the end of the proceedings)

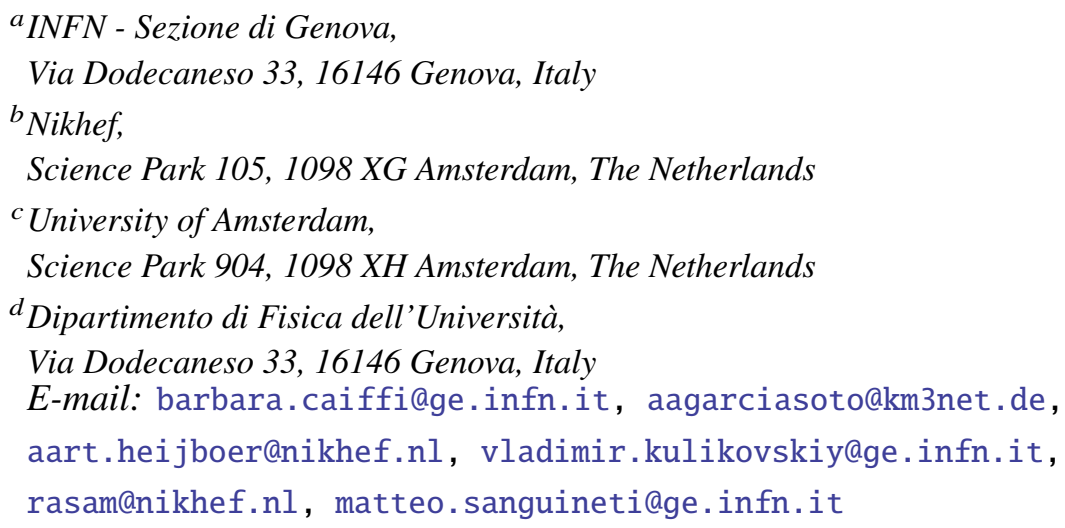

The identification of cosmic objects emitting high energy neutrinos could provide new insights about the Universe and its active sources. The existence of these cosmic neutrinos has been proven by the IceCube collaboration, but the big question of which sources these neutrinos originate from, remains unanswered. The KM3NeT detector for Astroparticle Research with Cosmics in the Abyss (ARCA), with a cubic kilometer instrumented volume, is currently being built in the Mediterranean Sea. It will excel at identifying cosmic neutrino sources due to its unprecedented angular resolution for muon neutrinos $\left(<0.2^{\circ}\right.$ for $E>10 \mathrm{TeV}$ neutrinos). KM3NeT has a view of the sky complementary to IceCube, and is sensitive to neutrinos across a wide range of energies. In order to identify the signature of cosmic neutrino sources in the background of atmospheric neutrinos and muons, statistical methods are being developed and tested with Monte-Carlo pseudoexperiments. This contribution presents the most recent sensitivity estimates for diffuse, point-like and extended neutrino sources with KM3NeT/ARCA.

\footnotetext{
*** 37th International Cosmic Ray Conference (ICRC2021), ***

*** 12-23 July $2021 * * *$

*** Berlin, Germany - Online ***
}

\footnotetext{
${ }^{*}$ Presenter
} 


\section{Introduction}

In 2013, the IceCube Neutrino Observatory confirmed the existence of a high-energy astrophysical neutrino flux [1]. However, the origin of most of these neutrinos remains unknown. The KM3NeT/ARCA detector [2] that is currently under construction at the bottom of the Mediterranean Sea, will consist of 2 building blocks, each one comprising 115 lines with 18 digital optical modules per line. It will have an excellent pointing resolution $\left(<0.2^{\circ}\right.$ for $E>10 \mathrm{TeV}$ muon neutrinos), it will be sensitive in a large energy range $(\mathrm{GeV}-\mathrm{PeV})$, with a sky coverage for upgoing neutrinos complementary to the IceCube detector and includes the galactic centre.

In order to identify a cosmic neutrino signal on top of the atmospheric background of muons and neutrinos, statistical methods are being developed based on Monte Carlo pseudo experiments. Compared to previously published work [3], the new methods presented here are the start of a bigger software framework for all future high energy astrophysical analysis. After applying the right cuts to increase the signal to background ratio, the detector response functions are determined. These distributions, like the so called effective area, the energy resolution and angular resolution are converted to probability density functions and are used as an input for the unbinned likelihood analysis to calculate the expected sensitivity of KM3NeT/ARCA to diffuse, point source and extended sources in our universe. The methods, and results will be presented in this contribution.

\section{Method}

\subsection{Simulations used}

The analysis is based on GENHEN (version v7r6) [4] Monte Carlo data simulations of $10^{2}-10^{8}$ $\mathrm{GeV}$ neutrino interactions for the KM3NeT/ARCA detector. Neutrinos are weighted using either cosmic or atmospheric flux models. For the latter, the conventional flux of Honda(2006) [5] (with Gaidder-H3a [6] knee-correction is used) is complemented by a prompt component parametrisation [7] with Gaidder-H3a knee-correcction. The $v_{\tau}$ channel is currently not taken into account. Atmospheric muons are simulated by the Mupgage package (version v3r4he) [8]. Events are subjected to a full simulation of light generation and to the response of the detector, and are processed through the same trigger and track reconstruction algorithms that are foreseen for data.

\subsection{Up-going and horizontal track selection}

Aim The analysis uses tracks reconstructed as horizontal or upward going $\left(\theta<100^{\circ}\right)$. A first set of well-reconstructed events are selected using requirements on, amongst others, reconstruction likelihood and the angular error estimate. On the events that are not selected a boosted decision tree (BDT) is applied. Events with a high BDT score are added to the sample as well. The BDT was trained using 20 variables related to the reconstructed track. Because the background contribution is larger for horizontal tracks, two different values are chosen to apply the cut for up-going (BDT $>0$ ) and horizontal tracks (BDT > 0.7).

Performance The overall performance of the selection as described in this section is presented in Table 1 and Figures 1, and 2. Table 1 shows the number of events with up-going and horizontal 


\begin{tabular}{l|l|r|r} 
& & Zenith cut & Total \\
\hline muons & all & 208685.6 & 249.5 \\
\hline conv. $v$ & all & 54012.4 & 42744.4 \\
& $+\mathrm{w} /$ muon & 49937.4 & 42687.7 \\
& $+\alpha<10^{\circ}$ & 43480.8 & 41454.4 \\
\hline prompt $v$ & all & 225.3 & 128.5 \\
& $+\mathrm{w} /$ muon & 147.9 & 127.6 \\
& $+\alpha<10^{\circ}$ & 129.3 & 124.4 \\
\hline $\operatorname{cosmic} v$ & all & 158.7 & 99.4 \\
& $+\mathrm{w} /$ muon & 113.5 & 98.6 \\
& $+\alpha<10^{\circ}$ & 101.2 & 97.0
\end{tabular}

Table 1: Number of expected events for one year of operation with one building block after selecting events with horizontal or up-going tracks (third column) and passing the overall selection (fourth column).
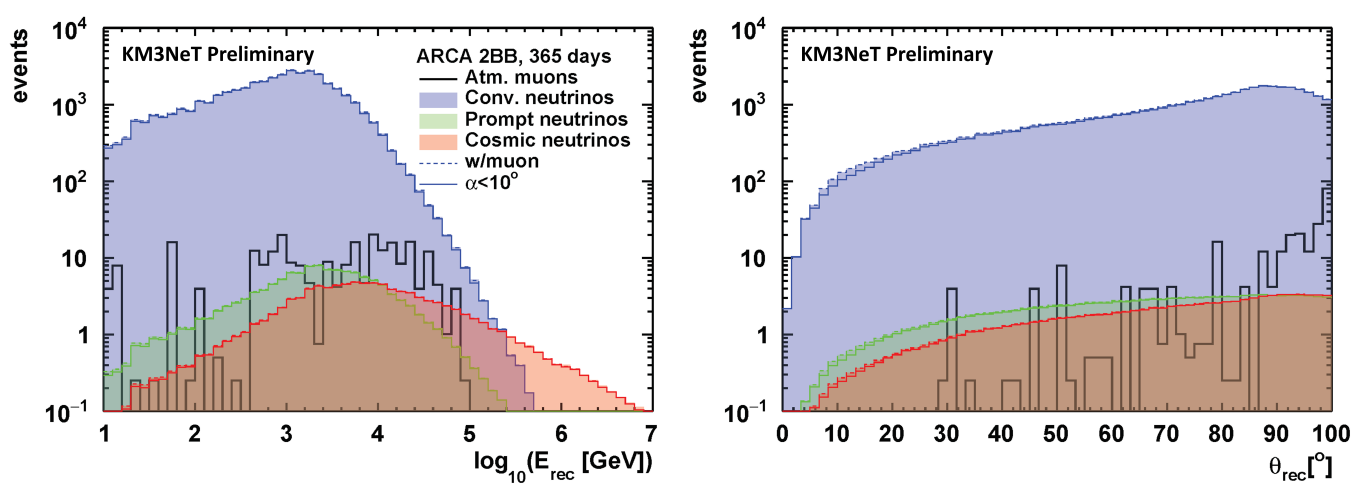

Figure 1: Energy (left) and zenith angle (right) distributions for reconstructed up-going or horizontal tracks passing the signal-like or the BDT cut.

tracks, and those events that fulfill the signal-like criteria, or survive the BDT cut. The final sample contains both these type of events.

The neutrino purity of the final sample, defined as the ratio between the number of selected neutrino events and the number of selected events $=99.4 \%$, of which $99.9 \%$ are $v_{\mu}$. The signal efficiency, defined as the ratio between the number of selected signal events and number of signal events with up-going or horizontal tracks, is computed for each neutrino component independently as a function of the neutrino energy. Overall the efficiency for well reconstructed tracks is above $90 \%$ and for events with muons in the detector above $80 \%$. One important feature of this selection is that the efficiency is almost identical for neutrino fluxes with very different shapes. Hence, the BDT selection will not be biased towards a certain cosmic flux assumption.

Instrument response functions From the selected event sample detector response functions are created, and are used as an input for the likelihood analysis. The detector response is assumed to be uniform in azimuth angle. Variations with zenith angle and energy (also in resolution) are taken into account. 

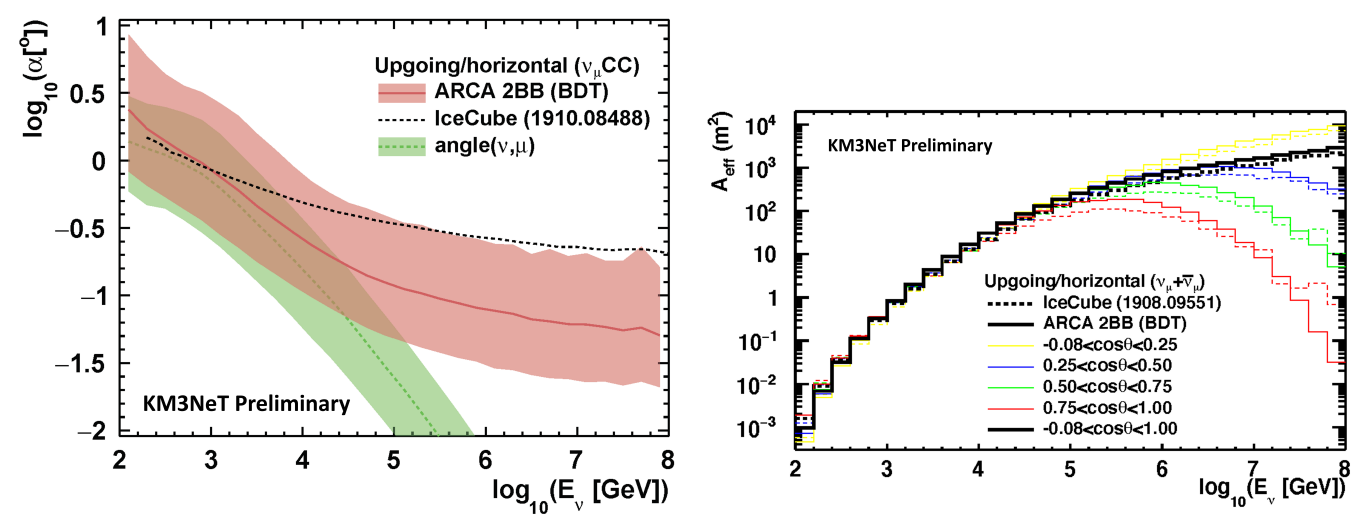

Figure 2: Left: Angular resolutions as function of the neutrino energy for $v_{\mu} \mathrm{CC}$ events. Icecube resolution was extracted from the point source analysis [9]. Right: Effective area as function of the neutrino energy for the selected event sample. Icecube effective area was extracted from the diffuse analysis [10].

\section{Likelihood formalism}

The analysis computes the extended maximum for the hypotheses with and without a cosmic neutrino source, to form the likelihood ratio as the optimal observable to test for the presence of a source. For this, a high-level description of the detector response is available in the form of tabulated (histogrammed) functions that describe the acceptance, the angular resolution and the performance of the energy estimate. Using the same probability density functions that are used to compute the event likelihoods, so-called pseudo-experiments (PE) can be generated. These represent full mock datasets of one or several years of real data.

The effective area as a function of energy $(E)$ and declination $(\delta)$ is computed by integrating over right ascension $(r)$

$$
A_{c, f}^{\mathrm{eff}}(E, \delta)=\frac{1}{2 \pi} \int A_{c, f}^{\mathrm{eff}}(E, \theta(\delta, r, t)) d r
$$

This is used to compute the total rate of observed events for a given flux:

$$
N^{\mathrm{tot}}(\delta)=\sum_{f \in \text { flavors }} \int d E A_{c, f}^{\mathrm{eff}}(E, \delta) \Phi_{f}(E) T,
$$

with $T$ the live time, and flavors refers here to $v_{\mu}$ and $\overline{\gamma_{\mu}}$ (although the can accommodate other flavours in the future)

\subsection{Point spread function}

The point spread function is available as a distribution of the angle $\alpha$, i.e. the angle between the true neutrino direction and the reconstructed one, as function of the true energy $E$ and $\cos (\theta)$. Two different methods with compatible results have been developed to smear the detector PSF to account for the source extension (in this analysis: 'extended PSF'). The first method is based on the random samplings. Sampling $\alpha$ from the detector PSF function and $\phi_{\mathrm{PSF}}$ in the range $[0,2 \pi]$ the reconstructed direction is build as $\left(\cos \phi_{\mathrm{PSF}} \sin \alpha, \sin \phi_{\mathrm{PSF}} \sin \alpha, \cos \alpha\right)$. The extended source 
neutrino direction is build in the same way with $\phi_{\text {ext }}$ sampled in the range $[0,2 \pi]$ and $\psi$ sampled from the source extension function: either flat disk $\left(\psi=R r^{1 / 2}\right)$ or Gaussian $\left(\psi=R(-2 \ln r)^{1 / 2}\right)$, where $r$ is a random number in a range $[0,1]$ and $R$ is the source extension. The angle between the reconstructed direction and the extended source neutrino direction, $\xi$ is then calculated. The extended PSF as $\xi$ distribution is build doing $10^{4}$ realisations.

In the second method the analytic convolution between the detector PSF and the source extension is used. For dihedral angle $\phi=\phi_{\mathrm{PSF}}-\phi_{\mathrm{ext}}$ one can evaluate:

$$
\cos \xi=\cos \alpha \cos \psi+\sin \alpha \sin \psi \cos \phi ; \quad \frac{d \phi}{d \xi}=\frac{1}{\sqrt{1-\cos \phi}} \frac{\sin \xi}{\sin \alpha \sin \psi} .
$$

This allows convolution over two angles $\alpha, \psi$ as follows:

$$
P(\xi)=\int_{0}^{\pi} \int_{|\alpha-\xi|}^{|\alpha+\xi|} P(\alpha) d \alpha P(\psi) d \psi P(\phi) \frac{1}{\sqrt{1-\cos \phi}} \frac{\sin \xi}{\sin \alpha \sin \psi} .
$$

Note that the integration over $\psi$ angle is done in a reduced range since the triangle rule holds for $\alpha, \psi, \xi$ on a sphere.

\subsection{Likelihood Function}

The global likelihood maximised is given by

$$
\mathcal{L}=\sum_{i \in \text { events }} \sum_{k \in \text { components }} \log \left[\frac{d N_{i, k}}{d \Omega d \log \left(E_{\text {rec }}\right)}\right]-N^{\text {tot }},
$$

where the concept of component has been introduced, to denote a physical contribution to the event rate (such as background, a diffuse flux, or a point-source flux of neutrinos). $N^{\text {tot }}$ denotes the total number of expected events in all flavors, and all channels. The quantity in square brackets is the likelihood of each event $i$ for the component in question. For atmospheric neutrino background, it is taken directly from a distribution of $\theta$ and $E_{\text {rec }}$ obtained from simulations of the backgrounds, for the channel corresponding to event $i$. For a point-source component,

$$
\frac{d N_{i}}{d \Omega d \log \left(E_{\mathrm{rec}}\right)}=\sum_{f \in \text { flavors }} \int d E \frac{d P_{f c}(E, \theta, \alpha)}{d \Omega d \log \left(E_{\mathrm{rec}}\right)} A_{f, c}^{\mathrm{eff}}(E, \theta) \Phi_{f}(E) .
$$

Here, for each event, the probability density function $P$ takes into account both the energy- and angular resolution, which are assumed to factorise.

\subsection{Pseudo-experiment generation}

Background events are generated by randomly drawing values of $E_{\text {rec }}$ and $\cos (\theta)$ from a 2-d distribution obtained with detailed simulations. For the generation of pseudo-experiments from a point-source, a 3-d histogram is produced, which contains the pdf of $\alpha$ and $E_{\text {rec }}$, and the time-of-day $\tau$. For a point source, the (sidereal) time-of-day uniquely determines the zenith angle (but not viseversa). The histogram is filled by computing the zenith angle from $\tau$ and the source coordinates. For each $\tau$, the number of events as function of $E_{\text {rec }}$ and $\alpha$ then follows from Eq. 6. From the resulting distribution, events are drawn to be included as signal events in pseudo-experiments. 


\section{Results}

The analysis tools described in the previous sections are used to evaluate the sensitivity of the KM3NET detector to diffuse, point-like and extended neutrino sources with KM3NeT/ARCA. Here our main results are presented.

Diffuse flux analysis In this section the sensitivity to characterise the astrophysical neutrino flux measured by IceCube's diffuse analysis [17] is presented. To constrain the parameters associated with the flux model, confidence intervals will be constructed using the profile likelihood technique applying Wilks' theorem.

The Asimov dataset [18] is generated assuming a nominal conventional flux, zero prompt flux and single power law spectra for the cosmic flux $\left(\Phi=0.963 \cdot 10^{-6} E^{-2.37}\left[\mathrm{GeV}^{-1} \mathrm{~cm}^{-2} \mathrm{~s}^{-1} \mathrm{sr}^{-1}\right]\right)$. Currently, three normalisation parameters $\left(\Phi_{\text {conv }}, \Phi_{\text {prompt }}\right.$ and $\left.\Phi_{0}\right)$ and the spectral slope of the cosmic flux $(\gamma)$ are fitted. Additional nuisance parameters associated with the systematic uncertainties on the atmospheric predictions or detector response are not incorporated yet.

In Figure 3 the expected sensitivity of KM3NeT/ARCA is compared with the IceCube result assuming similar lifetimes. On the one hand, the narrower contours of this analysis are mainly due to the lack of systematic uncertainties. On the other hand, the more elongated shape is due to a different modelling of the prompt component in the IceCube analysis [16] and this analysis [7].
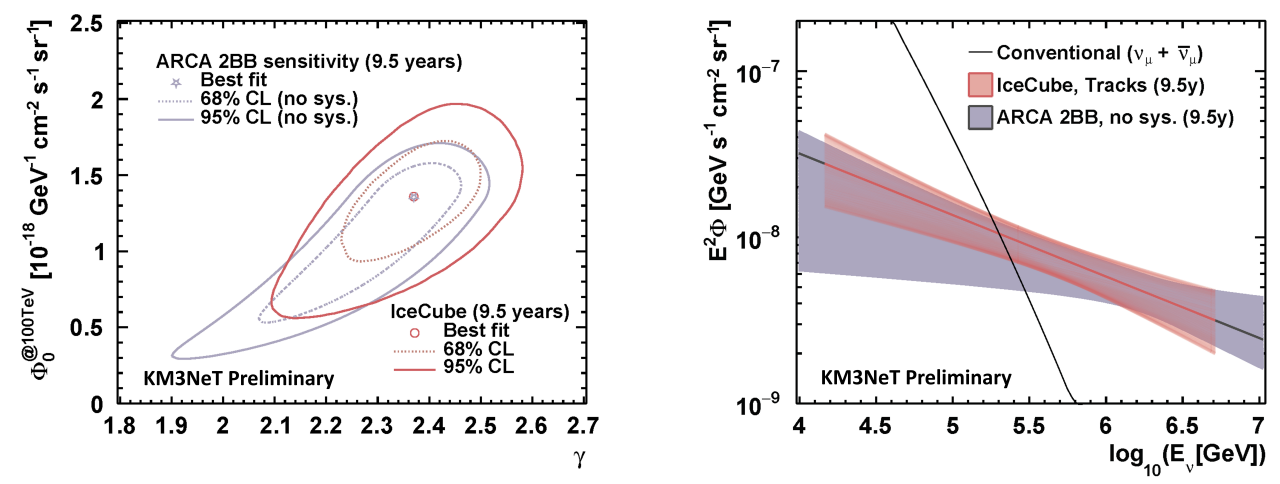

Figure 3: Left: Two-dimensional confidence regions of the astrophysical parameters. Right: Neutrino spectra for the diffuse flux. Grey band represents the sensitivity estimates of the $68 \%$ confidence interval using 9.5years of KM3NeT/ARCA (no systematics are included). The red band represents the best fit result from the IceCube analysis [17]. The black line is the conventional atmospheric neutrino flux prediction [5].

$E^{-2}$ point source analysis The sensitivity of $\mathrm{KM} 3 \mathrm{NeT}$ to point sources with an $E^{-2}$ flux is analysed for different sky positions, and for 3, and 7 years of data taking. Using the likelihood formalism, the source strength added to the background sample is varied between 0 and 20 events per year per building block. From the corresponding H1 distributions (4000 pseudo experiments), and the median of the H0 distribution (40.000 pseudo experiments) the number of signal events needed for a $90 \% \mathrm{CL}$ exclusion is determined. The two flux normalisation parameters $\left(N_{\mathrm{bkg}}, N_{\text {sig }}\right.$ are fitted in the analysis. The spectral slope of the cosmic flux $(\gamma=2)$ is kept fixed. In Figure 4 the results are shown in comparison to similar studies for 13 years of data taking with ANTARES (see ICRC poster contribution: poster 1142 ), and 7 years for IceCube [14]. 

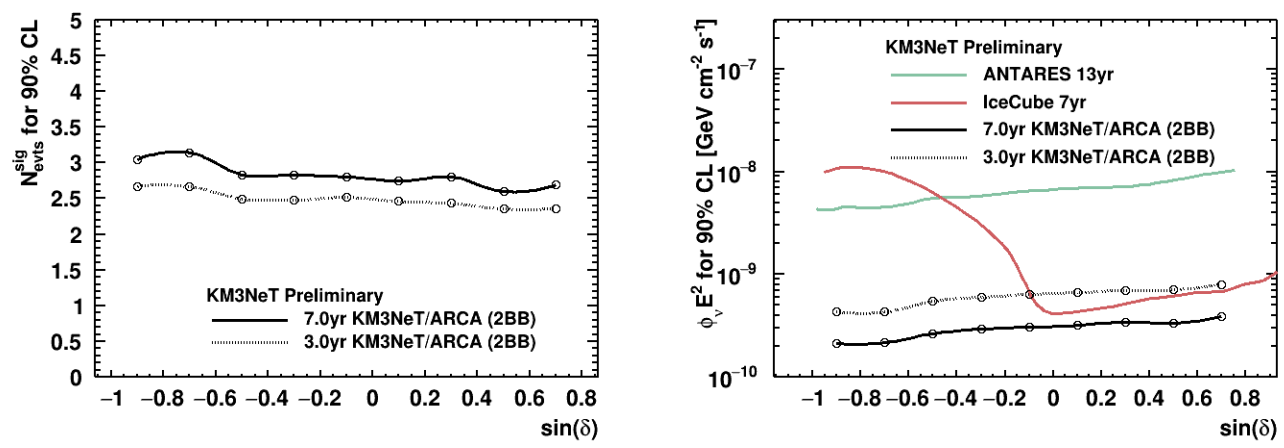

Figure 4: Number of events (left) and fluxnormalisation (right) for $90 \%$ CL limits to detect a $E^{-2}$ point source with KM3NeT/ARCA in comparison with ANTARES 13 years (see ICRC poster contribution: poster 1142 ), and IceCube 7 years [14].
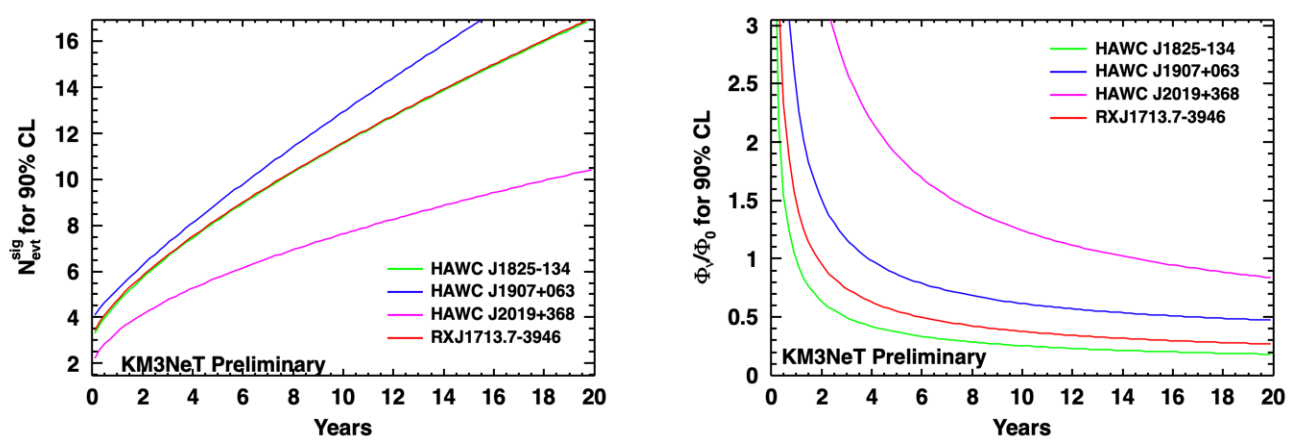

Figure 6: Left: Average upper limit at 90\% CL. Right: Sensitivity at 90\% CL.

Extended source analysis The sensitivity of KM3NeT to the sources in Table 2 is estimated. The prescriptions in [15] is implemented to derive the expected neutrino flux, as shown in Figure 5.

\begin{tabular}{|c|c|c|c|}
\hline Source & $\begin{array}{c}\text { Decl, RA } \\
{[\mathrm{deg}]}\end{array}$ & $\begin{array}{c}\text { ext } \\
{[\mathrm{deg}]}\end{array}$ & Ref. \\
\hline $\begin{array}{c}\text { RXJ } \\
1713.7-3946\end{array}$ & $\begin{array}{c}-39.77 \\
258.8\end{array}$ & $\begin{array}{c}0.60 \\
\text { (disk) }\end{array}$ & {$[11]$} \\
\hline HAWC & -13.37 & 0.53 & {$[12]$} \\
J1825-134 & 276.4 & (Gauss) & \\
\hline HAWC & 36.76 & 0.356 & {$[13]$} \\
J2019+368 & 304.92 & (Gauss) & \\
\hline HAWC & 6.32 & 0.67 & {$[12]$} \\
J1907+063 & 286.91 & (Gauss) & \\
\hline
\end{tabular}

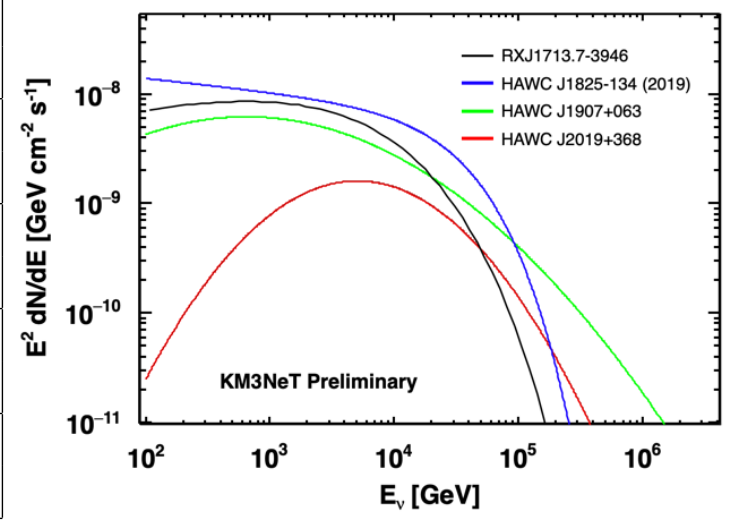

Table 2: Gamma source parameters. The label Figure 5: Expected neutrino fluxes of the sources (100\% disk/Gauss is the assumed source morphology. hadronic scenario is assumed).

The results are summarised in Figure 6. This study demonstrates the capability of the KM3NeT detector to achieve a $90 \%$ CL sensitivity for 3 of the 4 considered sources in less than 4 years; for the most promising source (HAWC J1825-134) the sensitivity is achieved approximately in 1 year. 


\section{Conclusion and discussion}

We have shown that our new likelihood method for KM3NeT to do high energy sensitivity studies for diffuse, point-like and extended neutrino source is in place, working, and providing convincing first results for KM3NeT/ARCA detector.

\section{References}

[1] IceCube Collaboration, Science 342.6161 (2013).

[2] KM3NeT Collaboration, J. Phys. G: Nuclear and Particle Physics 43.8 (2016): 084001.

[3] KM3NeT Collaboration, Astroparticle Physics 111 (2019): 100-110.

[4] Bailey D.J. (Ph.D. thesis) University of Oxford, United Kingdom (2002) http://antares.in2p3.fr/

[5] M. Honda et al., Phys.Rev. D75 (2007) 043006.

[6] T. Gaisser, Astropart.Phys. 35 (2012) 801-806.

[7] R. Enberg et al., Phys.Rev. D78 (2008) 043005.

[8] G. Carminati et al., Comput. Phys. Commun. 179, (2008) 915.

[9] M. Aartsen et al., Phys. Rev. Lett. 124, 051103 (2020).

[10] M. Aartsen et al., PoS-ICRC2019-1017 (2019).

[11] F. Aharonian et al. (H.E.S.S. Coll.), Astron. Astrophys. 464 (2007) 235.

[12] A. Abeysekara at al. (HAWC Coll.), Phys. Rev. Lett. 124 (2020) 2, 021102.

[13] A. Albert et al. (HAWC Coll.), Astrophys. J. 911 (2021) 2, 143

[14] IceCube Collaboration., Astrophys. J. 835.2 (2017) 151.

[15] F. L. Villante, F. Vissani, Phys. Rev. D 78 (2008) 103007.

[16] A. Fedynitch et al., EPJ Web Conf. 99 (2015) 08001.

[17] N. Whitehorn (IceCube Collaboration), NuTel Conference (2021).

[18] G. Cowan at al., EPJ C 71 (2011) 1554. 


\section{Full Author List: KM3NeT Collaboration}

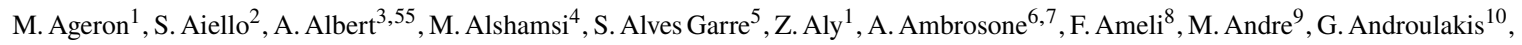
M. Anghinolfi ${ }^{11}$, M. Anguita ${ }^{12}$, G. Anton ${ }^{13}$, M. Ardid ${ }^{14}$, S. Ardid ${ }^{14}$, W. Assal ${ }^{1}$, J. Aublin ${ }^{4}$, C. Bagatelas ${ }^{10}$, B. Baret ${ }^{4}$, S. Basegmez du Pree ${ }^{15}$, M. Bendahman ${ }^{4,16}$, F. Benfenati ${ }^{17,18}$, E. Berbee ${ }^{15}$, A. M. van den Berg ${ }^{19}$, V. Bertin ${ }^{1}$, S. Beurthey ${ }^{1}$, V. van Beveren ${ }^{15}$, S. Biagi ${ }^{20}$, M. Billault ${ }^{1}$, M. Bissinger ${ }^{13}$, M. Boettcher ${ }^{21}$, M. Bou Cabo ${ }^{22}$, J. Boumaaza ${ }^{16}$, M. Bouta ${ }^{23}$, C. Boutonnet $^{4}$, G. Bouvet ${ }^{24}$, M. Bouwhuis ${ }^{15}$, C. Bozza ${ }^{25}$, H.Brânzass ${ }^{26}$, R. Bruijn ${ }^{15,27}$, J. Brunner ${ }^{1}$, R. Bruno ${ }^{2}$, E. Buis ${ }^{28}$, R. Buompane ${ }^{6,29}$, J. Busto ${ }^{1}$, B. Caiffi ${ }^{11}$, L. Caillat ${ }^{1}$, D. Calvo ${ }^{5}$, S. Campion ${ }^{30,8}$, A. Capone ${ }^{30,8}$, H. Carduner ${ }^{24}$, V. Carretero ${ }^{5}$, P. Castaldi ${ }^{17,31}$, S. Celli ${ }^{30,8}$, R. Cereseto ${ }^{11}$, M. Chabab ${ }^{32}$, C. Champion ${ }^{4}$, N. $\mathrm{Chau}^{4}$, A. Chen ${ }^{33}$, S. Cherubinini ${ }^{20,34}$, V. Chiarella ${ }^{35}$, T. Chiarusi ${ }^{17}$, M. Circella ${ }^{36}$, R. Cocimano ${ }^{20}$, J. A. B. Coelho ${ }^{4}$, A. Coleiro ${ }^{4}$, M. Colomer Molla ${ }^{4,5}$, S. Colonges ${ }^{4}$, R. Coniglione ${ }^{20}$, A. Cosquer ${ }^{1}$, P. Coyle ${ }^{1}$, M. Cresta ${ }^{11}$, A. Creusot ${ }^{4}$, A. $\mathrm{Cruz}^{37}$, G. Cuttone ${ }^{20}$, A. D’Amico ${ }^{15}$, R. Dallier ${ }^{24}$, B. De Martino ${ }^{1}$, M. De Palma ${ }^{36,38}$, I. Di Palma ${ }^{30,8}$, A. F. Díaz ${ }^{12}$, D. Diego$\operatorname{Tortosa}^{14}$, C. Distefano ${ }^{20}$, A. Domi ${ }^{15,27}$, C. Donzaud ${ }^{4}$, D. Dornic ${ }^{1}$, M. Dörr ${ }^{39}$, D. Drouhin ${ }^{3,55}$, T. Eberl ${ }^{13}$, A. Eddyamoui ${ }^{16}$, T. van Eeden ${ }^{15}$, D. van Eijk ${ }^{15}$, I. El Bojaddaini ${ }^{23}$, H. Eljarrari ${ }^{16}$, D. Elsaesser ${ }^{39}$, A. Enzenhöfer ${ }^{1}$, V. Espinosa ${ }^{14}$, P. Fermani ${ }^{30,8}$, G. Ferrara ${ }^{20,34}$, M. D. Filipović ${ }^{40}$, F. Filippini ${ }^{17,18}$, J. Fransen ${ }^{15}$, L. A. Fusco ${ }^{1}$, D. Gajanana ${ }^{15}$, T. Gal ${ }^{13}$, J. García Méndez ${ }^{14}$, A. Garcia Soto $^{5}$, E. Garçon ${ }^{1}$, F. Garufi ${ }^{6,7}$, C. Gatius ${ }^{15}$, N. Geißelbrecht ${ }^{13}$, L. Gialanella ${ }^{6,29}$, E. Giorgio ${ }^{20}$, S. R. Gozzini ${ }^{5}$, R. Gracia ${ }^{15}$, K. Graf ${ }^{13}$, G. Grella ${ }^{41}$, D. Guderian ${ }^{56}$, C. Guidi ${ }^{11,42}$, B. Guillon ${ }^{43}$, M. Gutiérrez ${ }^{44}$, J. Haefner ${ }^{13}$, S. Hallmann ${ }^{13}$, H. Hamdaoui ${ }^{16}$, H. van Haren ${ }^{45}$, A. Heijboer ${ }^{15}$, A. Hekalo ${ }^{39}$, L. Hennig ${ }^{13}$, S. Henry ${ }^{1}$, J. J. Hernández-Rey ${ }^{5}$, J. Hofestädt ${ }^{13}$, F. Huang ${ }^{1}$, W. Idrissi Ibnsalih ${ }^{6,29}$, A. Ilioni ${ }^{4}$, G. Illuminati ${ }^{17,18,4}$, C. W. James ${ }^{37}$, D. Janezashvili ${ }^{46}$, P. Jansweijer ${ }^{15}$, M. de Jong ${ }^{15,47}$, P. de Jong ${ }^{15,27}$, B. J. Jung ${ }^{15}$, M. Kadler ${ }^{39}$, P. Kalaczyński ${ }^{48}$, O. Kalekin ${ }^{13}$, U. F. Katz ${ }^{13}$, F. Kayzel $^{15}$, P. Keller ${ }^{1}$, N. R. Khan Chowdhury ${ }^{5}$, G. Kistauri ${ }^{46}$, F. van der Knaap ${ }^{28}$, P. Kooijman ${ }^{27,57}$, A. Kouchner ${ }^{4,49}$, M. Kreter ${ }^{21}$, V. Kulikovskiy ${ }^{11}$, M. Labalme ${ }^{43}$, P. Lagier ${ }^{1}$, R. Lahmann ${ }^{13}$, P. Lamare ${ }^{1}$, M. Lamoureux $^{14}$, G. Larosa ${ }^{20}$, C. Lastoria ${ }^{1}$, J. Laurence ${ }^{1}$, A. Lazo $^{5}$, R. Le Breton ${ }^{4}$, E. Le Guirriec ${ }^{1}$, S. Le Stum ${ }^{1}$, G. Lehaut ${ }^{43}$, O. Leonardi ${ }^{20}$, F. Leone ${ }^{20,34}$, E. Leonora ${ }^{2}$, C. Lerouvillois ${ }^{1}$, J. Lesrel ${ }^{4}$, N. Lessing ${ }^{13}$, G. Levi ${ }^{17,18}$, M. Lincetto ${ }^{1}$, M. Lindsey Clark ${ }^{4}$, T. Lipreau ${ }^{24}$, C. LLorens Alvarez ${ }^{14}$, A. Lonardo ${ }^{8}$, F. Longhitano ${ }^{2}$, D. Lopez-Coto ${ }^{44}$, N. Lumb ${ }^{1}$, L. Maderer ${ }^{4}$, J. Majumdar ${ }^{15}$, J. Mańczak ${ }^{5}$, A. Margiotta ${ }^{17,18}$, A. Marinelli ${ }^{6}$, A. Marini ${ }^{1}$, C. Markou $^{10}$, L. Martin ${ }^{24}$, J. A. Martínez-Mora ${ }^{14}$, A. Martini ${ }^{35}$, F. Marzaioli ${ }^{6,29}$,

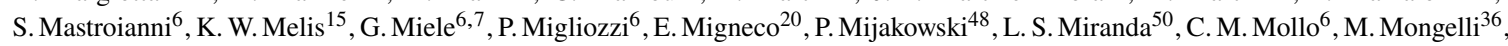
A. Moussa ${ }^{23}$, R. Muller ${ }^{15}$, P. Musico ${ }^{11}$, M. Musumeci ${ }^{20}$, L. Nauta ${ }^{15}$, S. Navas ${ }^{44}$, C. A. Nicolau ${ }^{8}$, B. Nkosi ${ }^{33}$, B. Ó Fearraigh ${ }^{15,27}$, M. O'Sullivan ${ }^{37}$, A. Orlando ${ }^{20}$, G. Ottonello ${ }^{11}$, S. Ottonello ${ }^{11}$, J. Palacios González ${ }^{5}$, G. Papalashviliite, R. Papaleo ${ }^{20}$, C. Pastore ${ }^{36}$, A. M. Păun ${ }^{26}$, G.E. Păvălaşş, G. Pellegrinini ${ }^{17}$, C. Pellegrino ${ }^{18,58}$, M. Perrin-Terrinin ${ }^{1}$, V. Pestel ${ }^{15}$, P. Piattelli ${ }^{20}$, C. Pieterse ${ }^{5}$, O. Pisanti ${ }^{6,7}$, C. Poirè ${ }^{14}$, V. Popa $^{26}$, T. Pradier $^{3}$, F. Pratolongo ${ }^{11}$, I. Probst ${ }^{13}$, G. Pühlhofer ${ }^{51}$, S. Pulvirenti ${ }^{20}$, G. Quéméner ${ }^{43}$, N. Randazzo ${ }^{2}$, A. Rapicavoli ${ }^{34}$, S. Razzaque $^{50}$, D. Real ${ }^{5}$, S. Reck ${ }^{13}$, G. Riccobene ${ }^{20}$, L. Rigalleau ${ }^{24}$, A. Romanov ${ }^{11,42}$, A. Rovelli ${ }^{20}$, J. Royon ${ }^{1}$, F. Salesa Greus ${ }^{5}$, D. F. E. Samtleben ${ }^{15,47}$, A. Sánchez Losa ${ }^{36,5}$, M. Sanguineti ${ }^{11,42}$, A. Santangelo ${ }^{51}$, D. Santonocito ${ }^{20}$, P. Sapienza ${ }^{20}$, J. Schmelling ${ }^{15}$, J. Schnabel ${ }^{13}$, M. F. Schneider ${ }^{13}$, J. Schumann ${ }^{13}$, H. M. Schutte ${ }^{21}$, J. Seneca ${ }^{15}$, I. Sgura ${ }^{36}$, R. Shanidze ${ }^{46}$, A. Sharma ${ }^{52}$, A. Sinopoulou ${ }^{10}$, B. Spisso ${ }^{41,6}$, M. Spurio ${ }^{17,18}$, D. Stavropoulos ${ }^{10}$, J. Steijger ${ }^{15}$, S. M. Stellacci ${ }^{41,6}$, M. Taiuti ${ }^{11,42}$, F. Tatone ${ }^{36}$, Y. Tayalati ${ }^{16}$, E. Tenllado ${ }^{44}$, D. Tézier ${ }^{1}$, T. Thakore ${ }^{5}$, S. Theraube ${ }^{1}$, H. Thiersen $^{21}$, P. Timmer $^{15}$, S. Tingay ${ }^{37}$, S. Tsagkli $^{10}$, V. Tsourapis ${ }^{10}$, E. Tzamariudaki ${ }^{10}$, D. Tzanetatos ${ }^{10}$, C. Valieri ${ }^{17}$, V. Van Elewyck ${ }^{4,49}$, G. Vasileiadis ${ }^{53}$, F. Versari ${ }^{17,18}$, S. Viola $^{20}$, D. Vivolo ${ }^{6,29}$, G. de Wasseige ${ }^{4}$, J. Wilms ${ }^{54}$, R. Wojaczyński ${ }^{48}$, E. de Wolf ${ }^{15,27}$, T. Yousfi ${ }^{23}$, S. Zavatarelli ${ }^{11}$, A. Zegarelli ${ }^{30,8}$, D. Zito ${ }^{20}$, J. D. Zornoza ${ }^{5}$, J. Zúñiga ${ }^{5}$, N. Zywucka ${ }^{21}$.

${ }^{1}$ Aix Marseille Univ, CNRS/IN2P3, CPPM, Marseille, France.

${ }^{2}$ INFN, Sezione di Catania, Via Santa Sofia 64, Catania, 95123 Italy.

${ }^{3}$ Université de Strasbourg, CNRS, IPHC UMR 7178, F-67000 Strasbourg, France.

${ }^{4}$ Université de Paris, CNRS, Astroparticule et Cosmologie, F-75013 Paris, France.

${ }^{5}$ IFIC - Instituto de Física Corpuscular (CSIC - Universitat de València), c/Catedrático José Beltrán, 2, 46980 Paterna, Valencia, Spain. ${ }^{6}$ INFN, Sezione di Napoli, Complesso Universitario di Monte S. Angelo, Via Cintia ed. G, Napoli, 80126 Italy.

${ }^{7}$ Università di Napoli “Federico II", Dip. Scienze Fisiche "E. Pancini”, Complesso Universitario di Monte S. Angelo, Via Cintia ed. G, Napoli, 80126 Italy.

${ }^{8}$ INFN, Sezione di Roma, Piazzale Aldo Moro 2, Roma, 00185 Italy.

${ }^{9}$ Universitat Politècnica de Catalunya, Laboratori d'Aplicacions Bioacústiques, Centre Tecnològic de Vilanova i la Geltrú, Avda. Rambla Exposició, s/n, Vilanova i la Geltrú, 08800 Spain.

${ }^{10}$ NCSR Demokritos, Institute of Nuclear and Particle Physics, Ag. Paraskevi Attikis, Athens, 15310 Greece.

${ }^{11}$ INFN, Sezione di Genova, Via Dodecaneso 33, Genova, 16146 Italy.

${ }^{12}$ University of Granada, Dept. of Computer Architecture and Technology/CITIC, 18071 Granada, Spain.

${ }^{13}$ Friedrich-Alexander-Universität Erlangen-Nürnberg, Erlangen Centre for Astroparticle Physics, Erwin-Rommel-Straße 1, 91058 Erlangen, Germany.

${ }^{14}$ Universitat Politècnica de València, Instituto de Investigación para la Gestión Integrada de las Zonas Costeras, C/ Paranimf, 1, Gandia, 46730 Spain.

${ }^{15}$ Nikhef, National Institute for Subatomic Physics, PO Box 41882, Amsterdam, 1009 DB Netherlands.

${ }^{16}$ University Mohammed V in Rabat, Faculty of Sciences, 4 av. Ibn Battouta, B.P. 1014, R.P. 10000 Rabat, Morocco.

${ }^{17}$ INFN, Sezione di Bologna, v.le C. Berti-Pichat, 6/2, Bologna, 40127 Italy.

\footnotetext{
${ }^{1}$ also at Dipartimento di Fisica, INFN Sezione di Padova and Università di Padova, I-35131, Padova, Italy
} 
${ }^{18}$ Università di Bologna, Dipartimento di Fisica e Astronomia, v.le C. Berti-Pichat, 6/2, Bologna, 40127 Italy.

${ }^{19}$ KVI-CART University of Groningen, Groningen, the Netherlands.

${ }^{20}$ INFN, Laboratori Nazionali del Sud, Via S. Sofia 62, Catania, 95123 Italy.

${ }^{21}$ North-West University, Centre for Space Research, Private Bag X6001, Potchefstroom, 2520 South Africa.

${ }^{22}$ Instituto Español de Oceanografía, Unidad Mixta IEO-UPV, C/ Paranimf, 1, Gandia, 46730 Spain.

${ }^{23}$ University Mohammed I, Faculty of Sciences, BV Mohammed VI, B.P. 717, R.P. 60000 Oujda, Morocco.

${ }^{24}$ Subatech, IMT Atlantique, IN2P3-CNRS, Université de Nantes, 4 rue Alfred Kastler - La Chantrerie, Nantes, BP 2072244307 France.

${ }^{25}$ Università di Salerno e INFN Gruppo Collegato di Salerno, Dipartimento di Matematica, Via Giovanni Paolo II 132, Fisciano, 84084 Italy.

${ }^{26}$ ISS, Atomistilor 409, Măgurele, RO-077125 Romania.

${ }^{27}$ University of Amsterdam, Institute of Physics/IHEF, PO Box 94216, Amsterdam, 1090 GE Netherlands.

${ }^{28}$ TNO, Technical Sciences, PO Box 155, Delft, 2600 AD Netherlands.

${ }^{29}$ Università degli Studi della Campania "Luigi Vanvitelli", Dipartimento di Matematica e Fisica, viale Lincoln 5, Caserta, 81100 Italy.

${ }^{30}$ Università La Sapienza, Dipartimento di Fisica, Piazzale Aldo Moro 2, Roma, 00185 Italy.

${ }^{31}$ Università di Bologna, Dipartimento di Ingegneria dell'Energia Elettrica e dell'Informazione "Guglielmo Marconi", Via dell’Università 50, Cesena, 47521 Italia.

${ }^{32}$ Cadi Ayyad University, Physics Department, Faculty of Science Semlalia, Av. My Abdellah, P.O.B. 2390, Marrakech, 40000 Morocco.

${ }^{33}$ University of the Witwatersrand, School of Physics, Private Bag 3, Johannesburg, Wits 2050 South Africa.

${ }^{34}$ Università di Catania, Dipartimento di Fisica e Astronomia "Ettore Majorana", Via Santa Sofia 64, Catania, 95123 Italy.

${ }^{35}$ INFN, LNF, Via Enrico Fermi, 40, Frascati, 00044 Italy.

${ }^{36}$ INFN, Sezione di Bari, via Orabona, 4, Bari, 70125 Italy.

${ }^{37}$ International Centre for Radio Astronomy Research, Curtin University, Bentley, WA 6102, Australia.

${ }^{38}$ University of Bari, Via Amendola 173, Bari, 70126 Italy.

${ }^{39}$ University Würzburg, Emil-Fischer-Straße 31, Würzburg, 97074 Germany.

${ }^{40}$ Western Sydney University, School of Computing, Engineering and Mathematics, Locked Bag 1797, Penrith, NSW 2751 Australia.

${ }^{41}$ Università di Salerno e INFN Gruppo Collegato di Salerno, Dipartimento di Fisica, Via Giovanni Paolo II 132, Fisciano, 84084 Italy.

${ }^{42}$ Università di Genova, Via Dodecaneso 33, Genova, 16146 Italy.

${ }^{43}$ Normandie Univ, ENSICAEN, UNICAEN, CNRS/IN2P3, LPC Caen, LPCCAEN, 6 boulevard Maréchal Juin, Caen, 14050 France.

${ }^{44}$ University of Granada, Dpto. de Física Teórica y del Cosmos \& C.A.F.P.E., 18071 Granada, Spain.

${ }^{45}$ NIOZ (Royal Netherlands Institute for Sea Research), PO Box 59, Den Burg, Texel, 1790 AB, the Netherlands.

${ }^{46}$ Tbilisi State University, Department of Physics, 3, Chavchavadze Ave., Tbilisi, 0179 Georgia.

${ }^{47}$ Leiden University, Leiden Institute of Physics, PO Box 9504, Leiden, 2300 RA Netherlands.

${ }^{48}$ National Centre for Nuclear Research, 02-093 Warsaw, Poland.

${ }^{49}$ Institut Universitaire de France, 1 rue Descartes, Paris, 75005 France.

${ }^{50}$ University of Johannesburg, Department Physics, PO Box 524, Auckland Park, 2006 South Africa.

${ }^{51}$ Eberhard Karls Universität Tübingen, Institut für Astronomie und Astrophysik, Sand 1, Tübingen, 72076 Germany.

${ }^{52}$ Università di Pisa, Dipartimento di Fisica, Largo Bruno Pontecorvo 3, Pisa, 56127 Italy.

${ }^{53}$ Laboratoire Univers et Particules de Montpellier, Place Eugène Bataillon - CC 72, Montpellier Cédex 05, 34095 France.

${ }^{54}$ Friedrich-Alexander-Universität Erlangen-Nürnberg, Remeis Sternwarte, Sternwartstraße 7, 96049 Bamberg, Germany.

${ }^{55}$ Université de Haute Alsace, 68100 Mulhouse Cedex, France.

${ }^{56}$ University of Münster, Institut für Kernphysik, Wilhelm-Klemm-Str. 9, Münster, 48149 Germany.

${ }^{57}$ Utrecht University, Department of Physics and Astronomy, PO Box 80000, Utrecht, 3508 TA Netherlands.

${ }^{58}$ INFN, CNAF, v.le C. Berti-Pichat, 6/2, Bologna, 40127 Italy. 